\section{SAFETY OF SUBSTRATE REDUCTION TERAPY IN A TODDLER WITH NIEMANN-PICK TYPE C DISEASE: A CASE REPORT}

J.S. Camelo, Jr. ${ }^{1}$, C.M. Lourenço², W. Marques, Jr. $^{2}$, M. Turcato², C. Funayama ${ }^{2}$, R. Sawamura ${ }^{1}$, M.M. Fernandes ${ }^{1}$

${ }^{1}$ Pediatrics, ${ }^{2}$ Neurology, University of São Paulo, Ribeirão Preto, Brazil

Background: Niemann-Pick type C (NPC) disease is a rare inborn error of metabolism caused by defective intracellular cholesterol traffic. Recently, substrate reduction therapy was introduced in the treatment of lysosomal storage disorders, especially of the glycosphingolipidoses with brain involvement. The iminosugar N-butyldeoxyjyrinomicin (miglustat) was reported to ameliorate neurologic phenotype of a murine NPC model. Initially used successfully in Gaucher and NPC diseases, concerns about its tolerance and safety arose related to peripheral neuropathy and diarrhea. The tolerance of very young children with NPC to miglustat is still largely unknown.

Case report: Female patient, 3 years old, born from healthy and non-consanguineous parents was referred to us at the age of one month with cholestasis, hepatomegaly and splenomegaly. Two older twin sisters died at 6 months with severe cholestasis with no diagnosis. Bone marrow biopsy revealed the presence of "foamy cells" and Gaucher and Niemann-Pick type A diseases were ruled out. However, chitotriosidase level was $1300 \mathrm{nmol} /$ $\mathrm{mL} / \mathrm{h}$, prompting a fibroblast culture from skin tissue that revealed a positive filipin staining and abnormal cholesterol esterification studies, confirming the diagnosis of NPC. Sequence analysis of NPC1 gene revealed a compound heterozygote, harboring a missense mutation (c.3104C>T) and a deletion of one nucleotide (c.3662delT).

Conclusions: Miglustat inhibits glucosylceramide synthase which catalyses the first step of glycosphingolipid synthesis. To the best of our knowledge, our patient is the youngest NPC patient treated during 36 months without any adverse event. This experience suggests that miglustat is well tolerated in small children and can ameliorate neurological phenotype.

\section{PROTECTIVE EFFECT OF COMPLEX LIPID MATRIX IN THE POSTNATAL DIET ON ADULT BODY FAT ACCUMULATION IS DEPENDENT ON LIPID-DROPLET SIZE}

\author{
E. van der Beek, A. Oosting, L. Schipper, \\ D. Kegler, M. Abrahamse
}

Danone Research, Wageningen, The Netherlands

We developed an IMF with a complex lipid matrix (CLM) which more closely resembles the physical properties of fat globules in breast milk, i.e. containing large lipid droplets coated with phospholipids. Postnatal exposure to infant milk formula (IMF) with CLM prevented excessive diet-induced fat accumulation in adult mice (Oosting et al., 2010). However, just increasing the lipid droplet size only marginally affected body composition development (Schipper et al., 2010). Hence, we investigated the contribution of the size of the lipid droplets in CLM on body composition development in mice.

Postnatally, between 16 and 42 days of age, male C57BI/6j mice were subjected to a diet containing standard IMF (CTR), CLM with lipid droplet size similar to CTR (CLM-S), or CLM (CLM-L). Subsequently, a moderate Western style diet (WSD, $10 \mathrm{w} \%$ fat) was fed until dissection at 98 days of age. Body composition was monitored by dual x-ray absorptiometry at 42,70 and 98 days of age.

Mice on both CLM diets showed less fat accumulation when challenged with the WSD during adulthood compared to the mice on CTR diet. However, the effect was more pronounced in mice on the CLM-L. In conclusion, although both phospholipid coating as well as increased size of lipid droplets in the postnatal diet protect against excessive fat accumulation when challenged with WSD in adulthood, combining both features enhances the beneficial effect significantly.

Oosting et al., 2010, EARNEST/EAPS

Schipper et al., 2010, EARNEST/EAPS 